\title{
Opinion: Airtightness for Decontamination by Fumigation of High-Containment Laboratories
}

Applied Biosafety:

Journal of ABSA International

2019, Vol. 24(4) 207-212

(C) ABSA International 2019

Article reuse guidelines:

sagepub.com/journals-permissions

DOI: $10.1177 / 153567601987 / 370$

journals.sagepub.com/home/apb

(B)AGE

\author{
Fanny Coppens ${ }^{\prime} \odot$, Nicolas Willemarck', and Didier Breyer ${ }^{\prime}$
}

\begin{abstract}
Introduction: While the European legislation states that laboratories of high-containment must be sealable for fumigation, they do not prescribe a minimal value for airtightness. Starting from a previous study in which we measured the airtightness in 4 BSL-3 laboratories with blower-door tests, we discuss the connection between airtightness and a successful decontamination by fumigation.

Methods: Biological indicators (Bls) consisting of spores of Geobacillus stearothermophilus on metal disks were laid out in laboratories of different levels of airtightness before performing a fumigation with aerosolized hydrogen peroxide using an automated device, according to the manufacturer's instructions.

Results: Incubation of all BI disks placed in the facility with the highest level of airtightness showed complete inactivation of spores. However, in the facility with a lower level of airtightness, not all spores were inactivated.

Discussion: Air leaks might be a factor in the outcome of the decontamination of a room by fumigation, as seen in the laboratory with a lower level of airtightness, but other factors associated with the fumigation process might also be critical for a successful decontamination.

Conclusion: We argue that a validation of the decontamination procedure, before first use or after important renovations of a laboratory of high-containment, is a more effective endpoint than reaching a predefined level of airtightness.
\end{abstract}

\section{Keywords}

airtightness, fumigation, decontamination, hydrogen peroxide, high-containment

European Directive 2009/41/EC ${ }^{1}$ on the contained use of genetically modified microorganisms states (Annex IV) that a laboratory of containment level 3 or 4 should be sealable (made airtight) for decontamination by fumigation and should be maintained at a negative pressure relative to the pressure of the immediate environment. Directive 2000/54/EC on the protection of workers from risks related to exposure to biological agents at work contains similar provisions (Annex V) concerning biological agents. Neither of those directives, nor the norms applicable in Belgium NBN EN 12738, 12128, or 12298 (Guidance for Containment of Animals Inoculated with Microorganisms in Experiments; Containment Levels of Microbiology Laboratories, Areas of Risk, Localities and Physical Safety Requirement; Biotechnology - Equipment - Guidance on Testing Procedures for Leaktightness, respectively), mention any required minimum numerical values for airtightness, although some other countries do. Decontamination by fumigation may be necessary in biocontainment facilities in several cases depending on risk assessment, including (1) after a bioincident with possible release of aerosols containing highly infectious agents (genetically modified or not), ${ }^{2}(2)$ at the end of any activity in which contamination of the biocontained area with highly infectious agents could not be avoided, (3) before maintenance, or (4) in the context of release for other purposes (decommissioning) of the facility and/or its equipment.

Fumigation is the method of choice to achieve a broadspectrum decontamination of a room. Surfaces that are difficult to reach can thereby be efficiently decontaminated. Several fumigants are available for decontamination, with formaldehyde, hydrogen peroxide (HP), and chlorine dioxide (CD) the most prevalent. The choice of the fumigant is often site specific because of the infrastructure itself (eg, the difficulty of maintaining room temperature and humidity or the sensitivity of equipment/devices to certain fumigants), because there is potential organic soiling that cannot be fully cleaned in advance, or because of cost considerations. ${ }^{3}$ Of the most commonly available fumigants, formaldehyde is the cheapest and

\footnotetext{
'Sciensano, Service Biosafety and Biotechnology, Brussels, Belgium

Corresponding Author:

Fanny Coppens, Sciensano, Service Biosafety and Biotechnology, Juliette Wytsmanstraat 14, 1050 Brussels, Belgium.

Email: fanny.coppens@sciensano.be
} 
easiest to set up, and it has been the most widely used fumigant. It is a true gas that spreads evenly within the available volume without the need for active circulation, similarly to $C D$ and contrary to HP: HP requires the use of a specific apparatus to circulate it, while CD breaks down very easily in sunlight and needs to be generated in situ. HP is the most sensitive to organic soiling, necessitating a thorough cleanup of all surfaces with detergent before decontamination. ${ }^{3}$ While formaldehyde and $\mathrm{CD}$ maintain some efficacy in the presence of organic matter such as blood or feces, a precleaning step is nevertheless always recommended. ${ }^{3}$ An important downside of formaldehyde is its toxicity and carcinogenicity, highlighting the importance of a high level of airtightness or sealability of the facility when formaldehyde is used as fumigant. As it is temperature sensitive, it must be used in a room where the temperature can be controlled to remain between 23 and $30^{\circ} \mathrm{C}^{3}$

A certain level of airtightness also benefits the effectiveness of the fumigation process by keeping the fumigant inside and in addition prevents the escape of harmful fumigants to adjoining rooms, with risk of exposure of the occupants. Therefore, it is important that the rooms that are to be decontaminated by fumigation can be made adequately airtight. Although the provisions of the EU legislation can be interpreted as requiring airtightness to be achieved only at the moment of the fumigation, it is preferable to achieve this airtightness as much as possible through the infrastructural quality of the building/ facility. Indeed, in case of an accidental release of highly infectious agents, procedures usually dictate that the room must be vacated and closed immediately, thereby rendering preparations prior to the cleanup and decontamination difficult or impossible.

The airtightness of a room can be quantified by means of a blower-door test (norm NBN EN 13829:2001, based on ISO 9972:1996), during which a pressure difference is created between the volume to be tested and the outside. A calibrated fan that is hermetically fitted in a (door) opening allows for the measurement of the leaked volume of air per hour, which is inversely proportional to the airtightness of the room. To effectively compare rooms of different sizes, the leaked volume of air per hour at the chosen pressure $\left(\mathrm{V}_{50}\right)$ is converted to the amount of air changes per hour (or the number of times per hour the total tested volume of air is leaked $\left[=n_{50}\right]$ in unit $\mathrm{h}^{-1}$ in case of a 50-Pa pressure difference), by normalizing against the total volume of the tested room.

Some countries do provide more numerical guidance in their norms or standards. For example, the applicable standard from Australia and New Zealand ${ }^{4}$ recommends a maximum air leakage rate of $10^{-5} \mathrm{~m}^{3} / \mathrm{Pa} . \mathrm{s}$, measured at an overpressure of 200 $\mathrm{Pa}$. The Canadian Biosafety Standards and Guidelines ${ }^{5}$ prescribe-depending on the work to be performed in the facility - a pressure decay test as a part of the commissioning (first certification) procedure for highly biocontained facilities, whereby the criteria for acceptance include 2 consecutive tests with a maximum of 250-Pa loss of pressure from an initial 500 $\mathrm{Pa}$ over a 20-minute period. Subsequent certifications, if no major modifications are performed to the secondary barrier, include visual inspection of the integrity of the containment barrier and tests with a smoke pencil or a tracer gas. ${ }^{5}$ In the United States, the Biosafety in Microbiological and Biomedical Laboratories manual does not include any numerical values for airtightness, stating only that all seams should be sealed and all openings should be sealable to facilitate space decontamination. This document recommends a pressure decay test only for newly constructed facilities of biosafety level 3 agriculture (BSL-3 Ag) for work with loose-housed animals, where the room is actually the primary containment. ${ }^{6}$

In a previous study, ${ }^{7}$ we reported airtightness measurements in 4 Belgian BSL-3 facilities. The airtightness at a pressure difference of $50 \mathrm{~Pa}$ was measured by the blower-door test in accordance with the requirements of NBN EN 13829:2001. One of the tested facilities was a newly built, state-of-the-art laboratory, while the others were renovations (a box-in-a-box setup, a research laboratory totally renovated with conventional building techniques, and a superficially renovated large-animal facility). Except for the laboratory, which was renovated with conventional building techniques and for which the airtightness test showed an air-change rate at $50 \mathrm{~Pa}\left(\mathrm{n}_{50}\right)$ of $4.08 \mathrm{~h}^{-1}$, the other labs showed a very similar performance in airtightness, with air-change rates of $0.8 \mathrm{~h}^{-1}$ or even better (for comparison purposes only, the airtightness standard for a passive house is set at $\left.\mathrm{n}_{50}=0.6 \mathrm{~h}^{-1}\right){ }^{8}$

These quantitative results triggered questions with respect to the qualitative aspect of the efficacy of decontamination through fumigation. Therefore, decontamination through fumigation with aerosolized hydrogen peroxide (aHP) using commercially available biological indicators (BIs) was performed in 2 of the tested facilities as a proof of principle: the facility with the lowest airtightness value $\left(\mathrm{n}_{50}=4.08 \mathrm{~h}^{-1}\right)$ was compared with one of the 3 others with similar values of airtightness $\left(\mathrm{n}_{50} \sim 0.8 \mathrm{~h}^{-1}\right)$, which represents in our view a typical situation of airtightness for a lab of high biological containment in Belgium. The experimental setup was kept as similar as possible between both facilities.

\section{Materials and Methods}

The fumigation by means of aHP was performed with a portable device (BioJet 200, adequate for rooms from 10 to 200 $\mathrm{m}^{3}$ ). It was filled with an $8 \% \mathrm{H}_{2} \mathrm{O}_{2}$ solution (Hydrogenium $\mathrm{C} 8$ ), and the volume of the room to be fumigated was entered as a parameter in the device, allowing it to operate for the calculated amount of time before switching off automatically. An additional 60-minute contact time was allowed after device switch off, before clearing out the remaining aHP via aeration through the heating, ventilation, and air-conditioning (HVAC) system for 40 minutes. The HVAC system was switched off during the fumigation and was restarted only at the end of the fumigant contact time.

The BIs were the APEX BI for gaseous HP, containing Geobacillus stearothermophilus spores (MesaLabs, Lakewood, $\mathrm{CO})$. One Tyvek pouch of BI contains 3 separate metal disks, coated with $10^{4}, 10^{5}$, and $10^{6}$ spores, respectively. The pouches 
Table I. Results of the Incubation of the BI Disks Placed in Various Locations in the Lab (Figure I) and Coated with $10^{4}, 10^{5}$, and $10^{6}$ Spores.

\begin{tabular}{|c|c|c|c|c|}
\hline & BI Location & $10^{4}$ & $10^{5}$ & $10^{6}$ \\
\hline I & In front of VHP device & Clear & Clear & Clear \\
\hline 2 & Cupboard under sink & Turbid & Turbid & Turbid \\
\hline 3 & Inside BSC & Clear & Clear & Turbid \\
\hline 4 & Top of BSC & Clear & Clear & Turbic \\
\hline 5 & Behind fridge & Clear & Clear & Turbid \\
\hline 6 & Anteroom (negative control) & Turbid & Turbid & Turbic \\
\hline 7 & $\begin{array}{l}\text { Inside plastic protective bag (negative } \\
\text { control) }\end{array}$ & Turbid & Turbid & Turbic \\
\hline
\end{tabular}

Abbreviations: BI, biological indicator; BSC, biosafety cabinet; VHP, vaporized hydrogen peroxide.

containing the BI were placed in triplicate in various locations of the laboratories, including behind or underneath large appliances, inside cupboards, and as far away from the fumigant generator as possible. After the fumigation experiment, each disc was placed in a sterile way in $10 \mathrm{~mL}$ tryptic soy broth (TSB) $30 \mathrm{~g} / \mathrm{L}$ (Oxoid, Hampshire, UK). To assess the sterility of the procedure of inoculation of TSB, 1 of 3 replicates was subjected to heat shock as per the manufacturer's recommendations, by placing the tubes for 15 minutes in a $90^{\circ} \mathrm{C}$ water bath before incubation (the heat shock aims to kill any potential contaminating - and temperature sensitive - bacteria that could have been introduced during the inoculation procedure while leaving the thermophile BI unaffected). The absence of a difference in outcome between the tubes subjected to heat shock or not for BI placed in the same spots in the laboratory indicated that the preparation of the culture tubes and their inoculation had indeed been performed in a sterile way. Incubation of all tubes was at $60^{\circ} \mathrm{C}$ under aerobic conditions with agitation for 48 hours. The readout of the viability of the recovered spores was performed visually by assessing whether the culture medium in the tubes showed turbidity. The successful decontamination of $G$ stearothermophilus with HP has already been published, although without mention of airtightness values of the rooms in which these were located. ${ }^{9-11}$

\section{Results and Discussion}

The facility with a previously tested airtightness value of $\mathrm{n}_{50} \sim$ $0.8 \mathrm{~h}^{-1}$, which was chosen for the fumigation tests with BI, is a box-in-a-box BSL-3 laboratory with a volume of $126 \mathrm{~m}^{3}$, consisting of a main area and 3 smaller dedicated working cells. It had been used for 10 years prior to the airtightness and fumigation tests. The BIs were placed at various locations throughout the common area of the lab and one working cell: inside and on top of a biosafety cabinet (BSC; longest distance from the fumigant generator), on top of a work bench, and underneath an incubator, as an example of a difficult-to-reach location. After the fumigation, performed as described in the "Materials and Methods" section, visual inspection of the growth medium

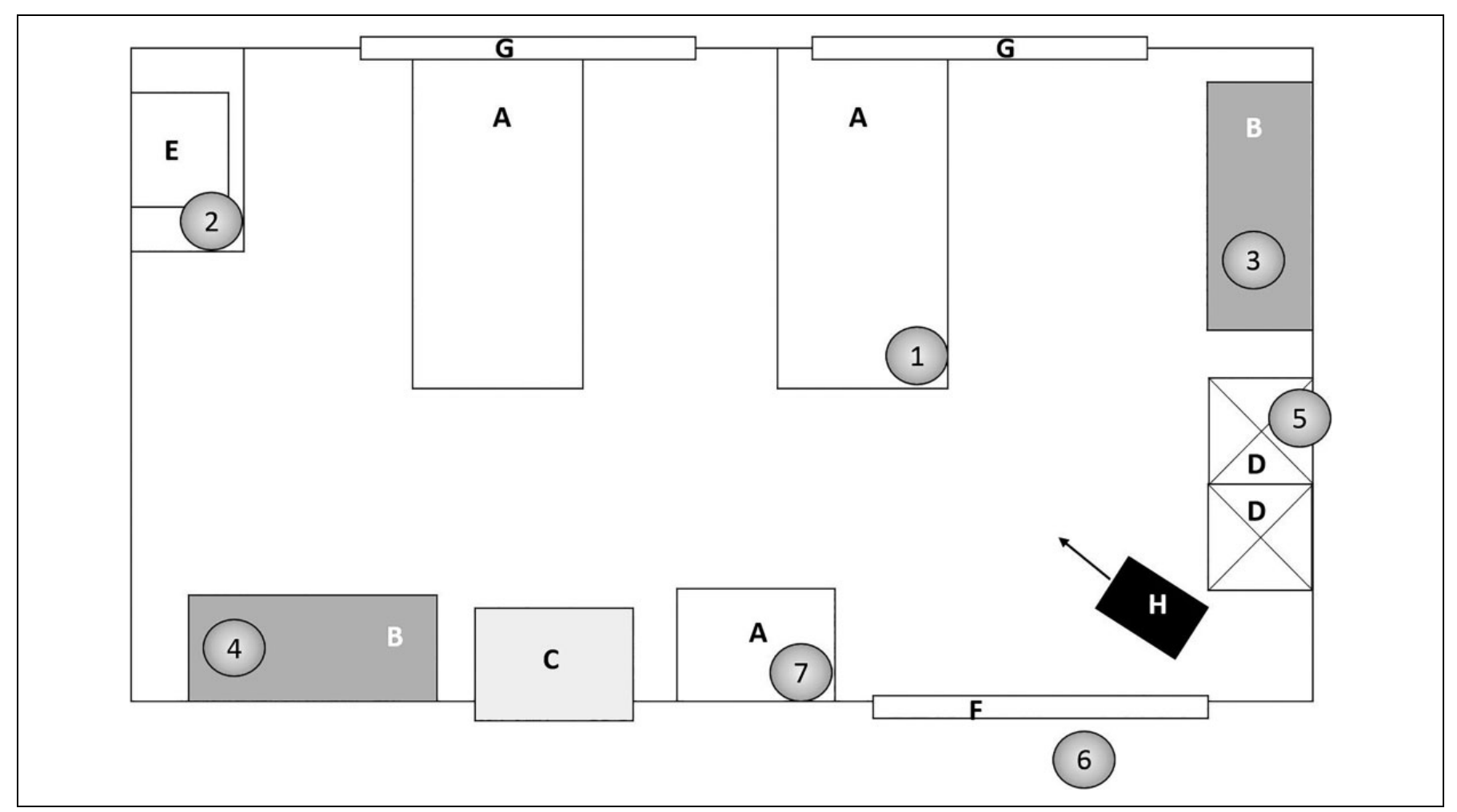

Figure I. Layout of the laboratory of $152 \mathrm{~m}^{3}$ during the fumigation. (A) Workbench. (B) Biosafety cabinet. (C) Double-door autoclave. (D) Fridge/freezer. (E) Sink. (F) Door. (G) Window. (H) Fumigation apparatus projecting the aerosolized hydrogen peroxide as indicated by the arrow. The numbers refer to the biological indicator placement as indicated in Table I. 
tubes after incubation at $60^{\circ} \mathrm{C}$ of the $\mathrm{BI}$ disks showed all the tubes had remained clear, except for the unexposed BI serving as a negative control. This indicated that the fumigation had resulted in a $6 \log _{10}$ reduction of the BI, which is generally considered the target for decontamination to be deemed complete.

On the other hand, after the fumigation performed in the facility with $\mathrm{n}_{50}=4.08 \mathrm{~h}^{-1}$, which is a single rectangular room with a volume of $152 \mathrm{~m}^{3}$, incubation of the disks coated with the BI in liquid culture medium resulted in several culture tubes that showed bacterial growth (Table 1; Figure 1). While the BI disks placed right in front of the aHP device (1) led to a clear culture medium after incubation, those placed in the closed cupboard under the sink (2), on the workbench (3), and on top (4) of the BSC and behind the fridge/freezer (5) showed a $<6 \log _{10}$ reduction of bacterial spores (Table 1). All controls showed the expected results: the tubes with empty medium remained clear (as did the stock bottle of liquid medium after many days at room temperature), and all the cultures from the pouches in the anteroom (6) and in the plastic protective bag (7), as well as BIs not exposed to the fumigant, showed turbidity. These results indicate that in this case, the decontamination of this facility was incomplete, even though the fumigant generator was adapted to the room's volume. They also showed that the distance of the BI to the fumigant generator and accessibility of the BI to the fumigant can influence the outcome of the inactivation of the BI. The cupboard inside which the BI was placed (2) was intentionally left closed, to simulate a difficult-to-reach location. The BIs behind the fridge/freezer (5) were placed in the 5- to $10-\mathrm{cm}$ gap with the wall, where the airflow caused by the heat generated by this fridge/freezer may have been an additional factor in disturbing the fumigant distribution. It is unclear why the inactivation of the BI placed on the BSC workbench (3) was incomplete, as this was located in quite close proximity to the fumigant generator, with the BSC turned off and the window left open. Further investigation in this lab at the time of the airtightness measurements revealed the frame between the double-door autoclave and the wall, the holes in the exterior wall for utility cables, and a sizeable gap in the ceiling's insulation to be important sources of air leaks. ${ }^{7}$

\section{Conclusions and Opinion}

Both decontaminations by fumigation described in this work have been performed according to the manufacturer's instructions with the same device, procedure, and fumigant. aHP was chosen because of its current wide use as fumigant, ease of use of the generating device, and absence of toxicity of the breakdown products. However, the aim of this article is not to discuss the ideal type of fumigant or fumigation setup; rather, the outcomes of the fumigation experiments merely serve as the starting point for the discussion. The rooms, for which the total fumigated volumes were similar and within the specifications for the device, were equipped with standard, nonporous laboratory furniture. The most apparent difference between both rooms was their airtightness, with the room with the lowest airtightness resulting in an unsuccessful decontamination procedure. Despite this, we argue that the incomplete decontamination of the BI might be linked not only to the airtightness of the room but also to inadequate fumigation parameters, such as the imperfect distribution of the fumigant. A complete decontamination of the BI might be obtained in a lab where the decontamination was not initially successful, for example, through the placement of additional fumigation devices, by repeating the fumigation process with the device in additional locations, or through the use of additional air-circulation devices.

In answer to the legal requirement of sealability for decontamination by fumigation, many applicable norms and guidance documents do not prescribe a minimal value for airtightness to be reached before first use of the facility. In our opinion, the important endpoint is the ability to decontaminate the facility by fumigation, and therefore, it is this process that should be validated. Validation of the fumigation process implies the successful decontamination of either BI or the most resistant of the microorganisms in use in the facility (in triplicate), using the fumigant and decontamination protocols that are applicable during the actual decontaminations. ${ }^{12,13}$ Measuring the airtightness of the room (by means of a blower-door test) can certainly be useful to obtain information on how effectively the airtightness has been implemented during the building or renovation of the facility. While it can provide a useful preliminary idea of the overall airtightness of the facility, this value is not enough for determining whether a facility can be sealable for decontamination by fumigation. While our results seem to suggest that rooms with a higher level of airtightness would be more likely to undergo an effective decontamination, this decontamination is, however, dependent on more factors than only the level of airtightness: the layout of the room and furniture, the temperature, humidity, presence of hot or cold spots, and the specificities of the fumigant and the apparatus all influence the outcome of the fumigation.

This raises the question of whether the fumigation process for decontamination should be validated prior to the first use of the facility. If this validation is indeed done successfully beforehand, the actual decontamination process by fumigation will allow for the facility to be quickly released again after the fumigation, thus leading to minimal operational downtime. If, however, the validation with $\mathrm{BI}$ is done only during the first actual decontamination of the facility, a negative validation result would lead to the need for the decontamination procedure to be repeated, which is time-consuming and leads to increased potential exposure of the workers to the biological agents present in the facility of high biological containment. This is especially impractical in cases in which the fumigation of a facility must be performed periodically and independently from the occurrence of a bioincident (for example, the decontamination of housing facilities for large animals after infection experiments).

Quantifying the airtightness of a room, once all the major building or renovation work has been carried out, will certainly yield valuable information. This numerical result will give an 
indication of the quality of the airtightness measures that were taken, and if the result is obtained before the finishing touches of the construction are applied, the contractor will have an easier time to search for and correct potential air leaks. The use of a smoke pencil is generally advised for finding leaks in the outer envelope of a room. It is difficult to predict the time during which the validation of the fumigation will remain valid, because of possible or expected degradation of the outer envelope of the facility. This should be taken into account through periodical renewed validation of the fumigation procedure, particularly following construction or renovation work that might have affected the airtightness of the room. In addition, HVAC parameters can be regularly monitored for variations (unexplained by the gradual saturation of the HEPA filters), which would suggest a decrease in airtightness. If this were the case, a renewed validation of the fumigation could be performed, preceded by a search for the potential air leaks in order to seal them.

The current European legal obligation leaves room for interpretation as to whether airtightness could be achieved only temporarily through special prepping of the room before the fumigation, but we would certainly not recommend this course of action. Rather, the intrinsic airtightness of a room (independent of possible air entry points for passive air flow, which are easily accessible for sealing) should be as high as possible, as the manual sealing of gaps before decontamination might be impossible or impractical (eg, after a bioincident, when access to the room before the decontamination is carried out, would represent an unnecessary risk to biological exposure). Furthermore, a high level of intrinsic airtightness not only minimizes the escape of the fumigant during the decontamination procedure and/or release of potential airborne infectious agents or aerosols but also results in a lower cost of day-to-day operation of the HVAC. ${ }^{3}$ In addition, a high intrinsic airtightness allows for a broader choice of possible fumigants.

In conclusion, we consider that, starting from the legal requirement for a facility of high biological containment to be "sealable for decontamination by fumigation," the successful validation of the decontamination procedure is the important endpoint. Since each facility has unique characteristics of room layout and airtightness, will be used for specific types of pathogenic (micro)organisms, and different fumigants may be used for decontamination, the validation requires a case-by-case approach, based on a correct risk assessment to minimize exposure to pathogen and fumigant. In our opinion, the validation could be seen as an important requirement of the regulatory authorization process prior to first use of the facility.

\section{Acknowledgments}

The authors wish to thank Aline Baldo, Emilie Descamps, Chuong Dai Do Thi, Amaya Leunda, and Katia Pauwels for their constructive review and comments, and the people in charge of the laboratories where the fumigation experiments were performed for their collaboration.

\section{Authors' Contributions}

Fanny Coppens and Nicolas Willemarck contributed equally to this work.

\section{Ethical Approval Statement}

Not applicable to this study.

\section{Statement of Human and Animal Rights}

Not applicable since no clinical studies or animal experimental results are included in this paper.

\section{Statement of Informed Consent}

Not applicable to this study.

\section{Declaration of Conflicting Interests}

The authors declared no potential conflicts of interest with respect to the research, authorship, and/or publication of this article.

\section{Funding}

The authors received no financial support for the research, authorship, and/or publication of this article.

\section{ORCID iD}

Fanny Coppens (D) https://orcid.org/0000-0001-6883-5889

\section{References}

1. Directive 2009/41/EC of the European Parliament and of the Council of 6 May 2009 on the contained use of genetically modified micro-organisms. (OJ L 125, 21.05.2009, p.75) This Directive repeals Directive 90/219/EEC and its successive following amendments: Directive 94/51/EC, Directive 98/81/EC and Decision 2001/204/EC.

2. Bennett A, Parks S. Microbial aerosol generation during laboratory accidents and subsequent risk assessment. J Appl Microbiol. 2006;100(4):658-663.

3. Gordon D, Carruthers B-A, Theriault S. Gaseous decontamination methods in high-containment laboratories. Appl Biosaf. 2012; 17(1):31-39.

4. AS-NZS 2243-3. Safety in laboratories: microbiological safety and containment. Sydney, Australia: Standards Australia; 2010.

5. Government of Canada. Canadian Biosafety Standard (CBS). 2nd ed. 2015. http://canadianbiosafetystandards.collaboration.gc.ca/ cbs-ncb/index-eng.php. Accessed April 2019.

6. Centers for Disease Control and Prevention. Biosafety in Microbiological and Biomedical Laboratories. 5th ed. Revised 2009. https://www.cdc.gov/biosafety/publications/bmb15/ index.htm.

7. Coppens F, Willemarck N, Herman P. Airtightness in Belgian High Biocontainment Facilities. https://www.biosafety.be/sites/ default/files/2016_coppens_willemarck_airtightnessreport.pdf. Published 2016. Accessed April 2019.

8. Passive House Institute. Criteria for the Passive House, EnerPHit and PHI Low Energy Building Standard, Version 9f, Revised 15. 
08.2016. https://passiv.de/downloads/03_building_criteria_en. pdf. Published 2016. Accessed April 2019.

9. Hall L, Otter JA, Chewins J, Wengenack NL. Use of hydrogen peroxide vapor for deactivation of Mycobacterium tuberculosis in a biological safety cabinet and a room. J Clin Microbiol. 2007; 45(3):810-815.

10. Holmdahl T, Lanbeck P, Wullt M, Walder MH. A head-to-head comparison of hydrogen peroxide vapor and aerosol room decontamination systems. Infect Control Hosp Epidemiol. 2011;32(9): 831-836.
11. Krishnan J, Berry J, Fey G, Wagener S. Vaporized hydrogen peroxide-based biodecontamination of a high-containment laboratory under negative pressure. Appl Biosaf. 2006;11(2): 74-80.

12. Beswick AJ, Farrant J, Makison C, et al. Comparison of multiple systems for laboratory whole room fumigation. Appl Biosaf. 2011;16(3):139-157.

13. Macellaro A, Karlsson L, Emmoth E, et al. Evaluation of biological indicator spores as tools for assessment of fumigation decontamination effectiveness. Appl Biosaf. 2015;20(4):183-191. 